\title{
Discovery of a new accreting millisecond X-ray pulsar in the globular cluster NGC 2808
}

\author{
A. Sanna ${ }^{1}$, A. Papitto ${ }^{2}$, L. Burderi ${ }^{1}$, E. Bozzo ${ }^{3}$, A. Riggio ${ }^{1}$, T. Di Salvo ${ }^{4}$, C. Ferrigno ${ }^{3}$, N. Rea ${ }^{5,6}$, and R. Iaria $^{4}$ \\ 1 Dipartimento di Fisica, Università degli Studi di Cagliari, SP Monserrato-Sestu km 0.7, 09042 Monserrato, Italy \\ e-mail: andrea.sanna@dsf.unica.it \\ 2 INAF, Osservatorio Astronomico di Roma, via di Frascati 33, 00044 Monteporzio Catone (Roma), Italy \\ 3 ISDC Data Centre for Astrophysics, Chemin d'Ecogia 16, 1290 Versoix, Switzerland \\ 4 Università degli Studi di Palermo, Dipartimento di Fisica e Chimica, via Archirafi 36, 90123 Palermo, Italy \\ 5 Anton Pannekoek Institute for Astronomy, University of Amsterdam, Postbus 94249, 1090 GE Amsterdam, The Netherlands \\ 6 Institute of Space Sciences (CSIC-IEEC), Campus UAB, Carrer Can Magrans s/n, 08193 Barcelona, Spain
}

Received 26 July 2016 / Accepted 8 November 2016

\begin{abstract}
We report on the discovery of coherent pulsations at a period of $2.9 \mathrm{~ms}$ from the X-ray transient MAXI J0911-655 in the globular cluster NGC 2808 . We observed X-ray pulsations at a frequency of $\sim 339.97 \mathrm{~Hz}$ in three different observations of the source performed with XMM-Newton and NUSTAR during the source outburst. This newly discovered accreting millisecond pulsar is part of an ultracompact binary system characterised by an orbital period of $44.3 \mathrm{~min}$ and a projected semi-major axis of $\sim 17.6$ lt-ms. Based on the mass function, we estimate a minimum companion mass of $0.024 M_{\odot}$, which assumes a neutron star mass of $1.4 M_{\odot}$ and a maximum inclination angle of $75^{\circ}$ (derived from the lack of eclipses and dips in the light-curve of the source). We find that the Roche-lobe of the companion star could either be filled by a hot $\left(5 \times 10^{6} \mathrm{~K}\right)$ pure helium white dwarf with a $0.028 M_{\odot}$ mass (implying $\left.i \simeq 58^{\circ}\right)$ or an old ( $>5 \mathrm{Gyr}$ ) brown dwarf with metallicity abundances between solar/sub-solar and mass ranging in the interval 0.065 to 0.085 $(16<i<21)$. During the outburst, the broad-band energy spectra are well described by a superposition of a weak black-body component $(k T \sim 0.5 \mathrm{keV})$ and a hard cut-off power-law with photon index $\Gamma \sim 1.7$ and cut-off at a temperature $k T_{\mathrm{e}} \sim 130 \mathrm{keV}$. Up until the latest Swift-XRT observation performed on 19th July, 2016, the source had been observed in outburst for almost 150 days, which makes MAXI J0911-655 the second accreting millisecond X-ray pulsar with outburst duration longer than 100 days.
\end{abstract}

Key words. X-rays: binaries - pulsars: general - stars: neutron - accretion, accretion disks - binaries: general

\section{Introduction}

Almost two decades after the discovery of the first accreting millisecond X-ray pulsar (SAX J1808.4-3658; Wijnands \& van der Klis 1998), the sample of accreting rapidly-rotating neutron stars (NS) harboured in low mass X-ray binary systems has increased in number up to 18 (see Burderi \& Di Salvo 2013; Patruno \& Watts 2012, for an extensive review on the topic). The extremely short spin periods shown by the accreting millisecond X-ray pulsars (AMXPs) are the result of long-lasting mass transfer from low-mass companion stars through an accretion disc onto a slow-rotating NS (also know as recycling scenario; Alpar et al. 1982). At the end of the mass transfer phase, a millisecond pulsar shining from the radio to the gamma-ray band, and powered by the rotation of its magnetic field, is expected to turn on. The close link shared by radio millisecond pulsars and AMXPs has been confirmed observationally by the transitional binary systems IGR J18245-2452 (Papitto et al. 2013b), PSR J0023+0038 (Archibald et al. 2009, 2015; Stappers et al. 2014; Patruno et al. 2014) and XSS J12270 (de Martino et al. 2010; Bassa et al. 2014; Roy et al. 2014; Papitto et al. 2015).

From the observation of the 15 AMXPs from which a state transition has not yet been observed (but see de Oña Wilhelmi et al. 2016), it can be highlighted that AMXPs have spin frequencies uniformly distributed in the range $\sim 180-600 \mathrm{~Hz}$. The orbital periods are always very short $\left(P_{\mathrm{orb}}<12 \mathrm{~h}\right)$, with the exception of the intermittent Aql X-1 that displays an orbital period of $\sim 18 \mathrm{~h}$ (Welsh et al. 2000). Five AMXPs of the sample show extremely short orbital periods $\left(P_{\text {orb }}<1 \mathrm{~h}\right)$ and can be considered ultra-compact binary systems. Orbital constraints allowed estimates of the typical mass values of the companion stars of AMXPs to be made, showing that very small donors are usually preferred (consistent with donor masses below $0.2 M_{\odot}$ ). Almost $80 \%$ of the sources in the sample show persistent X-ray pulsation during the outburst phase. The three remaining sources have been observed pulsating only occasionally: Aql X-1 (Casella et al. 2008), HETE J1900.1-2455 (Kaaret et al. 2006) and SAX J1748.9-2021 (Gavriil et al. 2007; Altamirano et al. 2008; Patruno et al. 2009a). The latter, for which the X-ray pulsations turned on and off intermittently during the outbursts, has recently been observed pulsating during its latest outburst (Sanna et al. 2016).

MAXI J0911-655 (also known as Swift J0911.9-6452) was first detected by the MAXI/GSC (Mihara et al. 2011) nova-alert system trigger on 19th February, 2016 (Serino et al. 2016), at a position compatible with the globular cluster NCG 2808. A few days later, on 29th February, 2016, the Burst Alert Telescope (BAT; Barthelmy et al. 2005) transient monitor onboard Swift detected X-ray activity within a region compatible with the previous MAXI/GSC observation, confirming the detection of a new X-ray transient source. The source position was determined by Swift (Kennea et al. 2016) and later on improved by Chandra 


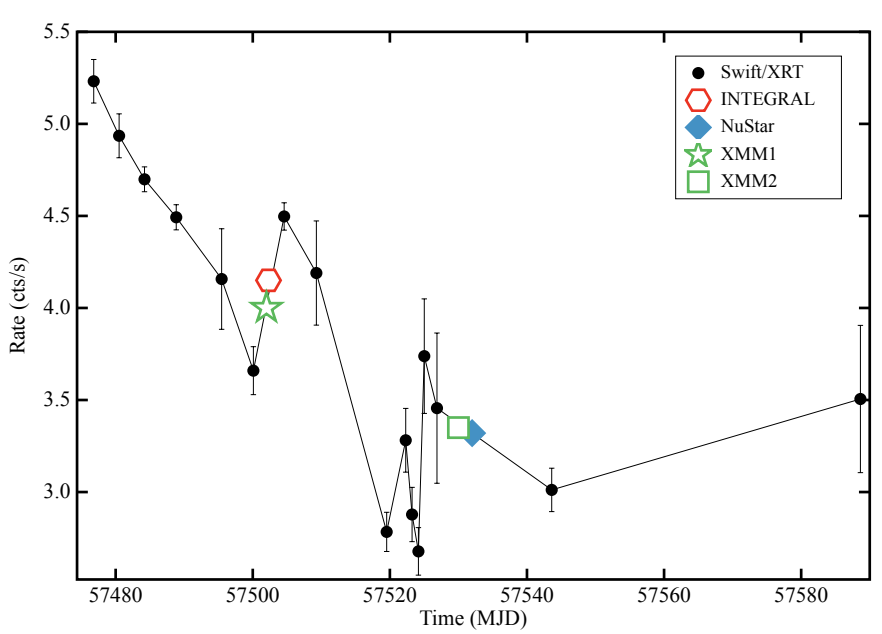

Fig. 1. Light-curve of the outburst of MAXI J0911-655 as observed by Swift-XRT (black points). Green symbols, blue diamond and red hexagon represent the observations collected by XMM-Newton, $\mathrm{NuS}$ $T A R$ and INTEGRAL, respectively.

(Homan et al. 2016). Here, we consider the most accurate source position, $R A=09^{\mathrm{h}} 12^{\mathrm{m}} 2.43^{\mathrm{s}}$ and $\mathrm{Dec}=-64^{\circ} 52^{\mathrm{m}} 6.4^{\mathrm{s}}$, determined by the latter with a $90 \%$ confidence level uncertainty of $0.6^{\prime \prime}$. Nearly-simultaneous radio and X-ray observations of the source performed by ATCA on 6th April, 2016, failed to detect a radio counterpart (Tudor et al. 2016). Here we report on the discovery of a coherent ms X-ray pulsation from MAXI J0911-655, and we describe the detailed analysis of $X M M-N e w t o n$ and NuSTAR observations from which we derived the orbital solution for the pulsar.

\section{Observations and data reduction}

\subsection{XMM-Newton}

We analysed the pointed XMM-Newton observations of MAXI J0911-655 performed on 24th April, 2016 (Obs. ID. 0790181401, hereafter XMM1) and on 22nd May, 2016, (Obs. ID. 0790181501, hereafter XMM2). During both EPIC observations the pn camera was operated in timing mode (with an exposure time of $\sim 38 \mathrm{ks}$ and $\sim 31 \mathrm{ks}$ for $X M M 1$ and $X M M 2$, respectively), while the RGS instrument was observing in spectroscopy mode. Figure 1 shows the light curve of observed outburst of the source monitored by Swift-XRT (black points). Each point represents the average count rate inferred from every pointed Swift-XRT observation. The mean count rate per observation have been extracted using the Swift-XRT on-line tool at http: //www. Swift. ac.uk (Evans et al. 2009). The green star and the green rectangle represent observations $X M M 1$ and $X M M 2$, respectively. For this analysis, we focused on the Epic-pn $(\mathrm{PN})$ data, which have both statistics and time resolution $(30 \mu \mathrm{s})$ required to investigate the time variability of the source. We performed the reduction of the PN data using the Science Analysis Software (SAS) v. 14.0.0 with the up-to-date calibration files, and adopting the standard reduction pipeline RDPHA (see Pintore et al. 2014, for more details on the method). We filtered the PN data in the energy range $0.3-10.0 \mathrm{keV}$, selecting events with PATTERN $\leq 4$ with single and double pixel pattern only. The observed PN mean count rate of the source extracted in the region RAWX range [31:45] during the observation XMM1 was $\sim 40 \mathrm{cts} / \mathrm{s}$, which decreased to $\sim 35 \mathrm{cts} / \mathrm{s}$ during the observation $X M M 2$. We estimated the background mean count rate in the RAWX range [2:6] to be $\sim 0.15 \mathrm{cts} / \mathrm{s}$ in the energy range $0.3-10 \mathrm{keV}$ for both observations. We verified that the background region was not heavily contaminated by the source. During the observations, no type-I burst episodes were recorded.

We corrected the PN photon arrival times for the motion of the Earth-spacecraft system with respect to the solar system barycentre by using the BARYCEN tool (DE-405 solar system ephemeris). We used the best available source position obtained from the Chandra identification of the source (Homan et al. 2016), and reported in Table 1.

For each PN event file, we extracted energy spectra setting "FLAG $=0$ " to retain only events optimally calibrated for spectral analysis. We generated response matrices and ancillary files using the RMFGEN and ARFGEN tools, respectively. We grouped the PN energy channels in order to have no more than three bins per energy resolution element and binned the energy spectra to have at least 25 counts per bin. We processed RGS data using the RGSPROC pipeline, extracting first and second order spectra and response matrices. We binned the RGS spectra in order to have at least 25 counts per bin.

\subsection{INTEGRAL}

INTEGRAL performed a dedicated target of opportunity observation of MAXI J0911-655 during the satellite revolution 1671, from 23rd April, at 23:43, until 25th April, 2016, at 6:51 (red hexagon in Fig. 1). The total exposure time collected in the direction of the source was $71.5 \mathrm{ks}$. INTEGRAL observations are divided into "science windows" (SCWs), that is, pointings with typical durations of $\sim 2-3 \mathrm{ks}$. As the observation was carried out in the hexagonal mode, the source was always located close to the aim point of all on-board instruments. All data were analysed with version 10.2 of the Off-line Scientific Analysis software (OSA) distributed by the ISDC (Courvoisier et al. 2003). We extracted the IBIS/ISGRI (Ubertini et al. 2003; Lebrun et al. 2003) mosaic in the 20-100 keV energy band and the JEM-X (Lund et al. 2003) mosaics in the $3-35 \mathrm{keV}$ energy band by using all data available. The source was detected in the ISGRI mosaic at a significance of $14 \sigma$ and a flux of $5.9 \pm 0.4 \mathrm{mCrab}^{1}$. This corresponds to approximately $1.1 \times 10^{-10} \mathrm{erg} \mathrm{cm}^{-2} \mathrm{~s}^{-1}$. Figure 2 shows the mosaicked IBIS/ISGRI image around the position of MAXI J0911-655, where the only other identifiable point source in the field of view is Cen X-3. In the JEM-X mosaic, the source was detected at $9 \sigma$ with a flux of $6.5 \pm 0.7 \mathrm{mCrab}$ (i.e. approximately $2.0 \times 10^{-10} \mathrm{erg} \mathrm{cm}^{-2} \mathrm{~s}^{-1}$ ).

As the source was relatively faint for INTEGRAL, we extracted a single spectrum for IBIS/ISGRI and the two JEM-X units by using all the available exposure time (the effective exposure time was of $44 \mathrm{ks}$ for ISGRI and $56 \mathrm{ks}$ for both JEM-X1 and JEM-X2). Due to the relatively low statistics of the INTEGRAL data we did not attempt a detailed timing analysis. JEM-X lightcurves with a time resolution of $2 \mathrm{~s}$ were extracted to search for type-I X-ray bursts, but no significant detection was found.

\subsection{NUSTAR}

MAXI J0911-655 was observed by NuSTAR (Obs. ID. 90201024002) on 24th May, 2016, (blue diamond in

\footnotetext{
1 The conversion between the upper limit on the source count-rate and flux has been done by using the observations of the Crab in revolution 1597, as described in Bozzo et al. (2016).
} 
Table 1. Orbital parameters and spin frequency of MAXI J0911-655 obtained from the analysis of the XMM-Newton observations of the source.

\begin{tabular}{|c|c|c|c|c|}
\hline Parameters & $X M M 1$ & $X M M 2$ & $X M M 1+X M M 2$ & NuSTAR \\
\hline RA (J2000) & \multicolumn{4}{|c|}{$09^{\mathrm{h}} 12^{\mathrm{m}} 2.43^{\mathrm{s}}$} \\
\hline $\operatorname{Dec}(\mathrm{J} 2000)$ & \multicolumn{4}{|c|}{$-64^{\circ} 52^{\mathrm{m}} 6.4^{\mathrm{s}}$} \\
\hline Orbital period $P_{\text {orb }}(\mathrm{s})$ & 2659.71(14) & $2659.93(7)$ & $2659.93312(47)$ & $a$ \\
\hline Projected semi-major axis $x$ (lt-s) & $0.01759(2)$ & $0.017598(11)$ & $0.017595(9)$ & $a$ \\
\hline Ascending node passage $T_{\mathrm{NOD}}(\mathrm{MJD})$ & $57502.185183(5)$ & $57530.600905(13)$ & $57502.185176(10)$ & $a$ \\
\hline Eccentricity $(e)$ & $<1.4 \times 10^{-2}$ & $<4 \times 10^{-3}$ & $<5 \times 10^{-3}$ & $a$ \\
\hline Spin frequency $v_{0}(\mathrm{~Hz})$ & $339.975071(3)$ & $339.9750123(3)$ & - & $339.974937(3)$ \\
\hline Spin frequency derivative $\dot{v}(\mathrm{~Hz} / \mathrm{s})$ & $-2.76(16) \times 10^{-9}$ & - & - & $-1.6(4) \times 10^{-10}$ \\
\hline Epoch of $v_{0}$ and $\dot{v}, T_{0}(\mathrm{MJD})$ & 57502.2 & 57530.6 & 57502.2 & 57532.0 \\
\hline$\chi_{v}^{2} /$ d.o.f. & $141.4 / 86$ & $85.9 / 108$ & $178.1 / 196$ & $22.4 / 9$ \\
\hline
\end{tabular}

Notes. Errors are at the $1 \sigma$ confidence level. The reported X-ray position of the source has a pointing uncertainty of 0.6" (see e.g. Homan et al. 2016). ${ }^{(a)}$ This parameter has been fixed to the value obtained from the combined analysis of the XMM-Newton observations.

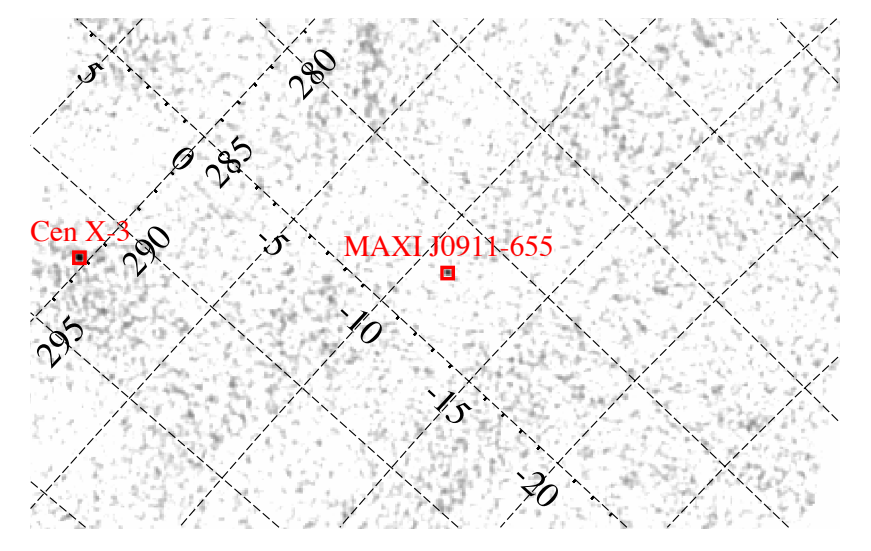

Fig. 2. Mosaicked IBIS/ISGRI image around the position of MAXI J0911-655 obtained from the observations performed in revolution $1671(20-100 \mathrm{keV})$. The source is detected in this mosaic at a significance of $14 \sigma$. Coordinates are expressed in degrees with respect to the galactic coordinate system.

Fig. 1). We performed standard screening and filtering of the events by means of the NUSTAR data analysis software (NUSTARDAS) version 1.5.1, resulting in an exposure time of approximately $60 \mathrm{ks}$ for each instrument. We selected source events extracting a circular region of radius $50^{\prime \prime}$ centered in the source position. We used the same extracting region, but centered far from the source, to extract the background. Spectra and light curves from each instrument were extracted and response files were generated using the NUPRODUCTS pipeline. Furthermore, we used the LCMATH task to generate background-subtracted light curves for the FMPA and FMPB, with an average count rate per instrument of $\sim 4$ counts/s. No type-I burst episode was recorded during the observation. We corrected the NUSTAR photon arrival times for the motion of the Earth-spacecraft system with respect to the solar system barycentre by using the BARYCORR tool (DE-405 solar system ephemeris). We used the best available source position reported in Table 1.

\section{Data analysis}

\subsection{Timing analysis}

We created a power density spectrum of the observation $X M M 1$ by averaging together power spectra produced over $512 \mathrm{~s}$ of data. We detected a highly significant $(13 \sigma)$ broad double-picked signal with frequency ranging between 339.96 and $339.99 \mathrm{~Hz}$. In order to obtain a first orbital motion solution for the NS, we inspected every 512 s-long power density spectrum for significant features in the $0.04 \mathrm{~Hz}$ interval around the frequency $339.98 \mathrm{~Hz}$. We modelled the detected spin frequency variation of the signal as the Doppler shift induced by the binary orbital motion:

$\Delta_{v}=\Delta_{v_{0}}-\frac{2 \pi v_{0} x}{P_{\text {orb }}} \cos \left(\frac{2 \pi\left(t-T_{\mathrm{NOD}}\right)}{P_{\mathrm{orb}}}\right)$,

where $v_{0}$ is the spin frequency, $x$ is the projected semi-major axis of the NS orbit in light seconds, $P_{\text {orb }}$ is the orbital period, and $T_{\mathrm{NOD}}$ is the time of passage through the ascending node. We thus obtain a first estimate of the orbital ephemeris of the system, such as the orbital period $P_{\text {orb }}=2656(9) \mathrm{s}$, the projected semimajor axis $x=0.0175(7) \mathrm{lt}-\mathrm{s}, T_{\mathrm{NOD}}=57502.185(1)(\mathrm{MJD})$, as well as the spin frequency $v_{0}=339.9751(5) \mathrm{Hz}$.

Starting from this first timing solution, we corrected the photon time of arrivals of the XMM1 datasets for the delays caused by the binary motion applying the orbital parameters through the recursive formula

$t+z(t)=t_{\mathrm{arr}}$,

where $t$ is photon emission time, $t_{\text {arr }}$ is the photon arrival time to the solar system barycentre, $z(t)$ is the projection along the line of sight of the distance between the NS and the barycentre of the binary system in light seconds. For almost circular orbits (eccentricity $e \ll 1$ ) we can write

$z(t)=x \sin \left(\frac{2 \pi}{P_{\mathrm{orb}}}\left(t-T_{\mathrm{NOD}}\right)\right)$.

The correct emission times (up to an overall constant $D / c$, where $D$ is the distance between the solar system barycentre and the barycentre of the binary system) are calculated by iteratively solving the aforementioned Eq. (2), $t_{n+1}=t_{\text {arr }}-z\left(t_{n}\right)$, with $z(t)$ defined as in Eq. (3), with the conditions $D / c=0$, and $z\left(t_{n=0}\right)=0$. We iterated until the difference between two consecutive steps $\left(\Delta t_{n+1}=t_{n+1}-t_{n}\right)$ was lower than the absolute timing accuracy of the instrument used for the observations. In our case we set $\Delta t_{n+1}=1 \mu \mathrm{s}$.

We folded $300 \mathrm{~s}$ intervals of the XMM1 dataset into 16 phase bins around the estimate of the pulsar spin frequency reported above. We fitted each folded profile with a sinusoid in order to determine the corresponding sinusoidal amplitude and the fractional part of the epoch-folded phase residual. We considered 
only folded profiles for which the ratio between the amplitude of the sinusoid and its $1 \sigma$ uncertainty was larger than three. We attempted to fit the the folded profiles including a second harmonic component, but this component was significantly detected in less than $5 \%$ of the total number of intervals.

We modelled the temporal evolution of the pulse phase delays with the relation:

$\Delta \phi(t)=\phi_{0}+\Delta v_{0}\left(t-T_{0}\right)+\frac{1}{2} \dot{v}\left(t-T_{0}\right)^{2}+R_{\mathrm{orb}}(t)$,

where $T_{0}$ represents the reference epoch for the timing solution, $\Delta v_{0}=\left(v_{0}-\bar{v}\right)$ is the difference between the frequency at the reference epoch and the spin frequency used to epoch-fold the data, $\dot{v}$ is the spin frequency derivative, and $R_{\text {orb }}$ is the Roemer delay caused by the differential corrections to the set of orbital parameters used to correct the photon time of arrivals (see e.g. Deeter et al. 1981). If a new set of orbital parameters was found, photon time of arrivals were corrected using Eq. (3) and pulse phase delays were created and modelled with Eq. (4). We iterated this process until no significant differential corrections were found for the parameters of the model. Obtained best-fit parameters are shown in the first column of Table 1.

Using the aforementioned NS ephemeris, we corrected the photon time of arrivals of the second XMM-Newton observation (XMM2) by using Eq. (3). We calculated pulse phase delays over pulse profiles created folding $300 \mathrm{~s}$ data intervals fitted with a sinusoidal profile. No second harmonic component was required to model the pulse profiles. Using Eq. (4) we calculated the spin frequency and the best-fit orbital parameters for XMM2 (see second column of Table 1).

Uncertainties on the NS spin frequencies combined with the almost 30-day time gap between the two XMM-Newton observations did not allow us to phase connect the pulsations obtained from the two datasets. Still we were able to model together the phase delays induced by the orbital motion in the two observations. After correcting the photon time of arrivals of the $X M M 1$ and $X M M 2$ datasets using the orbital parameters determined from the timing analysis of $X M M 1$, we calculated pulse phase delays following the process described previously. We investigated differential correction to the adopted orbital parameters by fitting the pulse phase delays time evolution with the following models:

$$
\left\{\begin{array}{l}
\Delta \phi_{X M M 1}(t)=\sum_{n=0}^{3} \frac{C_{n}}{n !}\left(t-T_{0}\right)^{n}+R_{\mathrm{orb}}(t) \\
\Delta \phi_{X M M 2}(t)=\sum_{n=0}^{1} \frac{D_{n}}{n !}\left(t-T_{0}\right)^{n}+R_{\mathrm{orb}}(t)
\end{array},\right.
$$

where the first element of both equations represents a polynomial function used to independently model phase variations in each dataset. Additionally, the model includes a common residual orbital modulation component $R_{\mathrm{orb}}(t)$, which implies that the orbital parameters will be linked during the fit of the two datasets. This method has the advantage (with respect to modelling each observation separately) of improving the accuracy of the orbital parameters (e.g. the orbital period). Best-fit parameters are reported in Table 1, while in Fig. 3 we showed the pulse phase delays of the two observations with the best-fitting models (top panel), and the residuals with respect to the models.

In order to take into account the effect of the uncertainties regarding the source coordinates on the pulse phase delays, we used the expression of the residuals induced by the motion of the Earth for small variations of the source positions $\delta_{\lambda}$ and $\delta_{\beta}$ expressed in ecliptic coordinates $\lambda$ and $\beta$ (see, e.g.

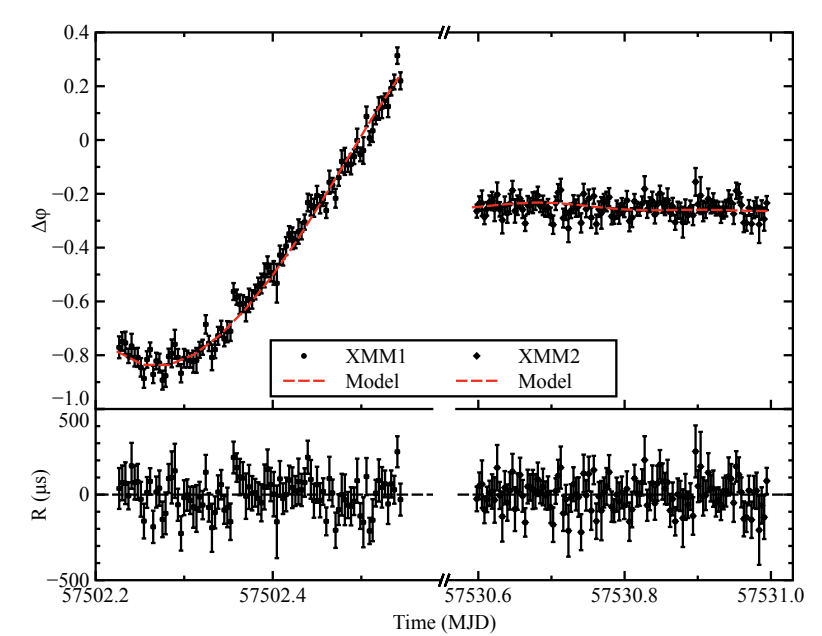

Fig. 3. Top panel: pulse phase delays as a function of time computed by epoch-folding $300 \mathrm{~s}$ data intervals of the XMM-Newton observations, together with the best-fit model (red dotted line, see text). Bottom panel: residuals in $\mu$ s with respect to the best-fitting orbital solution.

Lyne \& Graham-Smith 1990) to estimate the systematic uncertainties induced on the linear and quadratic terms of the pulse phase delays, which correspond to a systematic uncertainty in the spin frequency correction $\Delta v_{0}$, and the spin frequency derivative $\dot{v}$, respectively. The former corrections can be expressed as $\sigma_{v_{\text {pos }}} \leq v_{0} y \sigma_{\gamma}\left(1+\sin ^{2} \beta\right)^{1 / 2} 2 \pi / P_{\oplus}$, and $\sigma_{\dot{v}_{\text {pos }}} \leq v_{0} y \sigma_{\gamma}(1+$ $\left.\sin ^{2} \beta\right)^{1 / 2}\left(2 \pi / P_{\oplus}\right)^{2}$, where $y=r_{\mathrm{E}} / c$ is the semi-major axis of the orbit of the Earth in light-seconds, $P_{\oplus}$ is the Earth orbital period, $\sigma_{\gamma}$ is the positional error circle and $\beta$ corresponds to $\sim-70.7 \mathrm{de}-$ grees. Considering the $90 \%$ positional uncertainty of $0.6^{\prime \prime}$ reported by Homan et al. (2016), we estimated $\sigma_{v_{\text {pos }}} \leq 9 \times 10^{-8} \mathrm{~Hz}$, and $\sigma_{\dot{\nu}_{\mathrm{pos}}} \leq 2 \times 10^{-14} \mathrm{~Hz} / \mathrm{s}$, respectively. We added in quadrature these systematic uncertainties to the statistical errors of $v_{0}$, and $\dot{v}$ reported in Table 1 .

Finally, using the binary parameters inferred from the timing analysis of the combined XMM-Newton observations, we corrected the photon arrival times of the NUSTAR observation. We look for pulsations performing epoch-folding searches of the whole observation using 16 phase bins. Starting from the spin frequency measured during the observation XMM2 (closest in time) we explored the frequency space with steps of $10^{-7} \mathrm{~Hz}$ for a total of 5001 steps. We detected a significant X-ray pulsation at a mean frequency of $v_{\text {nus }}=339.974925(3) \mathrm{Hz}^{2}$, which significantly differs from the starting seed spin frequency. To investigate the timing properties of the source during the NuSTAR observation, we calculated the pulse phase delays epoch-folding time intervals of approximately $6000 \mathrm{~s}$ (shortest time interval required to detect a significant pulse profile). We modelled each epoch-folded pulse profile with a sinusoid in order to determine the corresponding sinusoidal amplitude and the fractional part of phase residual, no second harmonic was required to model the profile. The length of the pulse profiles compared with the orbital binary period clearly excludes the possibility of investigating differential correction of the set of orbital parameters used to correct the photon arrival times. We therefore modelled the pulse phase delays with a polynomial function of the second order to investigate the NS spin frequency time evolution. Best-fit parameters are shown in Table 1.

2 The error on the spin frequency value has been estimated by means of Monte Carlo simulations. 


\subsection{Spectral analysis}

The goal of the spectral analysis presented here is to characterise the X-ray emission of the newly discovered AMXP MAXI J0911-655 in a broad-band energy range. In order to do that we analysed the available X-ray observations of the source performed by different instruments, and we combined together observations close in time for which no spectral changes have been observed. We created two broad-band energy spectra, the first (Obs.1) includes the data collected by both the RGS and the PN instruments during the XMM1 observation, and the data collected by INTEGRAL that globally covered the time interval between 24th and 25th April, 2016. The second spectrum (Obs.2) includes the data collected by both the RGS and the PN instruments during the $X M M 2$ observation, and the data from the NUSTAR observation performed almost two days after XMM2.

Both broad-band spectra, reported in the top panel $(0.4-$ $100 \mathrm{keV}$ Obs.1) and bottom panel (0.4-80 keV Obs.2) of Fig. 4, appeared to be relatively hard. They are well fitted $\widetilde{\chi}_{v}^{2} /$ d.o.f. $1.04 / 1462$ and 1.05/2700, for Obs. 1 and Obs.2, respectively) by an absorbed power-law with high energy cutoffs, that proved statistically more significant than a simple power-law component $\left(\Delta \chi^{2}=26\right.$ and $\Delta \chi^{2}=360$ for the addition of one parameter, for Obs.1 and Obs.2, respectively). The component that takes into account interstellar absorption (TBABS) uses abundances and photoelectric cross-section tables from Anders \& Grevesse (1989) and Balucinska-Church \& McCammon (1992), respectively. We find a neutral column density $\left(N_{\mathrm{H}}\right)$ of $(0.25 \pm 0.01) \times$ $10^{22} \mathrm{~cm}^{-2}$ and $(0.29 \pm 0.01) \times 10^{22} \mathrm{~cm}^{-2}$, a photon index of $1.59 \pm$ 0.06 and $1.75 \pm 0.02$, and a cutoff temperature of $88_{-23}^{+120} \mathrm{keV}$ and $160_{-23}^{+32} \mathrm{keV}$, for Obs. 1 and Obs.2, respectively. Furthermore, we find evidence for a weak soft thermal component that we fitted with a blackbody of temperature $0.61 \pm 0.1 \mathrm{keV}$ in Obs. 1 , that decreases to $0.53 \pm 0.01 \mathrm{keV}$ for Obs.2. Finally, both spectra show the presence of a weak (marginally significant) and relatively narrow emission line in the range $6.5-6.6 \mathrm{keV}$, that we model with a gaussian profile with $\sigma$ ranging between 0.02 and $0.2 \mathrm{keV}$ and equivalent width between 11 and $16 \mathrm{eV}$, which we identified with $\mathrm{K} \alpha$ emission from Fe XXV.

We estimated the broad-band 0.4-100 keV absorbed (unabsorbed) flux of Obs. 1 and Obs. 2 to be $\sim 4.3 \times 10^{-10} \mathrm{erg} \mathrm{cm}^{-2} \mathrm{~s}^{-1}$ $\left(\sim 4.7 \times 10^{-10} \mathrm{erg} \mathrm{cm}^{-2} \mathrm{~s}^{-1}\right)$ and $\sim 3 \times 10^{-10} \mathrm{erg} \mathrm{cm}^{-2} \mathrm{~s}^{-1}(\sim 3.4 \times$ $\left.10^{-10} \mathrm{erg} \mathrm{cm}^{-2} \mathrm{~s}^{-1}\right)$, respectively. Considering a source distance of $\sim 9.5 \mathrm{kpc}$ (distance of the host globular cluster NGC 2808; Watkins et al. 2015) we estimate an unabsorbed luminosity of $\sim 5.1 \times 10^{36} \mathrm{erg} \mathrm{cm}^{-2}$, and $\sim 3.7 \times 10^{36} \mathrm{erg} \mathrm{cm}^{-2}$, for Obs. 1 and Obs.2, respectively, corresponding to $3 \%$ and $2 \%$ of the Eddington luminosity for accretion onto a standard $1.4 M_{\odot}$ NS.

\section{Discussion}

We discovered a new AMXP, MAXI J0911-655, which is located in the globular cluster NGC 2808. X-ray pulsations at $339.97 \mathrm{~Hz}$ were detected in the two XMM-Newton and in the NUSTAR observations performed during the outburst of the new $\mathrm{X}$-ray source, with an average pulse fraction of $7 \%$. We modelled the NS spin frequency drift shown by the source as Doppler shift induced by the binary orbital motion, finding an accurate orbital solution of the almost 45 min binary system. We found an accurate set of orbital parameters by fitting the pulse phase delays of the two XMM-Newton observations simultaneously, modelling the delays introduced by the orbital motion with a common set of parameters (orbitally phase-connected). On the other hand, the evolution with time of the NS spin frequency was kept independent between the two observations. The orbital
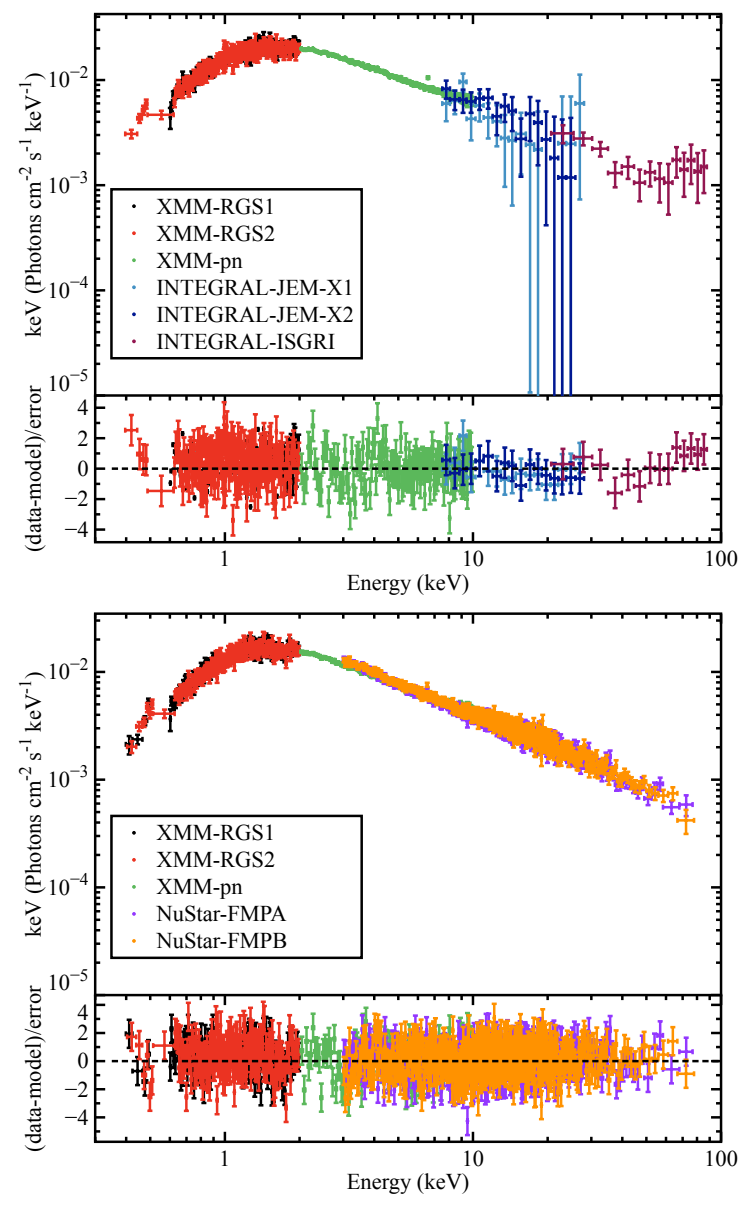

Fig. 4. Top panel: broad-band (0.4-100 keV) energy spectrum of Obs.1 including XMM-Newton RGS1 (black), RGS2 (red) and PN (green) and INTEGRAL JEM-X (blue and cyan) and IBIS/ISGRI. Bottom panel: broad-band (0.4-80 keV) energy spectrum of Obs.2 including XMM-Newton RGS1 (black), RGS2 (red) and PN (green) and NuSTAR FMPA/B (orange and violet). For both energy spectra the residuals with respect to the best fit models are given in units of $\sigma$.

period (44.3 $\mathrm{min})$ and the binary separation (17.6 lt-ms) clearly illustrate the ultra-compact nature of the binary system, which recalls very similarly the orbital properties of other AMXPs such as XTE J1751-305 (Markwardt et al. 2002; Papitto et al. 2008; Riggio et al. 2011), XTE J0299-314 (Galloway et al. 2002), XTE J1807-294 (Kirsch et al. 2004; Riggio et al. 2008; Chou et al. 2008; Patruno et al. 2010b), SWIFT J1756.9-2508 (Krimm et al. 2007; Linares et al. 2008; Patruno et al. 2010a) and NGC 6440 X-2 (Altamirano et al. 2010; Bult et al. 2015).

As reported in Table 1, both XMM1 and NUSTAR observations required a very large spin-down NS frequency derivative in order to adequately model the evolution of the pulse phase delays. We note that both measurements are artefacts caused by instrumental issues of the satellites during the observations; more specifically the XMM1 observations showed anomalies on the Spacecraft Time Correlation file which were only partially corrected by the XMM-Newton science team (XMM-Newton team, priv. comm.), while the NUSTAR observation suffered from a time drift of the internal clock of the instrument (Madsen et al. 2015). The presence of spurious spin frequency derivatives significantly changes the spin frequency measurements explaining the large discrepancy between the spin frequency detected during $X M M 2$ and the other two observations.

The latest Swift observation of MAXI J0911-655 performed on 19th July, 2016, showed X-ray activity comparable with that 
observed during the XMM-Newton and NUSTAR observations where the source has been observed to be pulsating. Assuming that the outburst started with the first detection of the source (19th February, 2016), we can estimate an outburst duration of at least 150 days, which is relatively long compared with the other AMXPs, although not unique. With the exception of the quasipersistent AMXP HETE J1900.1-2455 (observed in outburst for more than $8 \mathrm{yr}$ ), a long-lasting outburst has been observed for the ultra-compact AMXP XTE J1807-294, detected in outburst for almost 120 days during its 2003 outburst (Riggio et al. 2007). We note however, that the lack of high timing resolution observations at the extremes of the observed outburst prevent us from verifying the persistency of the X-ray pulsation during the long outburst.

The value of NS mass function $f\left(m_{2}, m_{1}, i\right) \sim 6.2 \times 10^{-6} M_{\odot}$ allows us to set constraints on the mass of the companion star. The lack of eclipses, as well as dips, in the light curve sets an upper limit $i \lesssim 75^{\circ}$ on the inclination angle (Frank et al. 2002), which provides a lower limit to the companion star of $m_{2} \gtrsim 0.024 M_{\odot}$ (assuming a $1.4 M_{\odot}$ NS), which increases up to $m_{2} \gtrsim 0.03 M_{\odot}$ if we consider a $2 M_{\odot}$ NS. Combining the condition for mass transfer via Roche-lobe overflow $\left(R_{2} \approx R_{L 2}\right)$ with the mass function, we can describe the companion mass radius as $R_{2} \simeq 0.2 m_{2}^{1 / 3} P_{\text {orb, } 1 \mathrm{~h}}^{2 / 3} R_{\odot}$, where $P_{\text {orb,h }}$ is the binary orbital period expressed in hours. In Fig. 5 we compare the companion mass-radius relation (black solid line) with detailed numericallysimulated mass-radius relations for low-mass hydrogen main sequence stars and brown dwarfs of different ages (black dotdashed line, $5 \mathrm{Gyr}$, and cyan dashed line, $10 \mathrm{Gyr}$; Chabrier et al. 2009) and different metallicity content (5 Gyr black dot-dashed line with solar metallicity $Z_{\odot}$ and sub-solar $\left(0.01 Z_{\odot}\right)$ abundances; Chabrier \& Baraffe 2000). From the intersections between mass-radius companion curve and the stellar models we can infer that the donor star is compatible with an old brown dwarf ( $\geq 5 \mathrm{Gyr}$ ), with mass in the interval 0.065 to $0.085 M_{\odot}{ }^{3}$ and metallicity abundances between solar and sub-solar. We note that age and metallicity content are compatible with estimates from deep IR studies of stellar populations of host cluster NGC 2808 (Massari et al. 2016). The latter companion mass range limits the binary inclination angle between 16 and 21 degrees. In Fig. 5 we also report theoretical mass-radius relations for cold $\left(10^{2} \mathrm{~K}\right)$ and hot $\left(5 \times 10^{6} \mathrm{~K}\right)$ pure helium white dwarfs (Deloye \& Bildsten 2003). The latter object is compatible with the mass-radius companion curve for a mass value of $\sim 0.028 M_{\odot}$, which implies an inclination angle of $\sim 58$ degrees. Studies on evolutionary paths should be investigated in order to understand which of the two proposed scenarios is more favourable.

Finally, the broad-band energy spectra of MAXI J0911-655 analysed here (Obs.1 and Obs.2) are well described by the superposition of a weak soft black-body-like component $(k T \sim$ $0.5 \mathrm{keV})$ and a hard high-energy cut-off power-law $(\Gamma \sim 1.7$ and $\left.k T_{\mathrm{e}} \sim 130 \mathrm{keV}\right)$, in agreement with spectral properties of other AMXPs observed in a hard state (Falanga et al. 2005a,b; Gierliński \& Poutanen 2005; Patruno et al. 2009b; Papitto et al. 2009, 2013a). Moreover, the source shows marginal evidence of a weak and narrow reflection component in the energy range $6.5-6.6 \mathrm{keV}$ which we identify as the $\mathrm{K} \alpha$ emission line from helium-like iron. The estimated broad-band

\footnotetext{
3 Mass values within the intersections, for which the Roche-lobe radius is larger than the estimated stellar radius, are still acceptable if we considered the possibility of the companion being bloated with respect to its thermal equilibrium radius because of irradiation from the compact object.
}

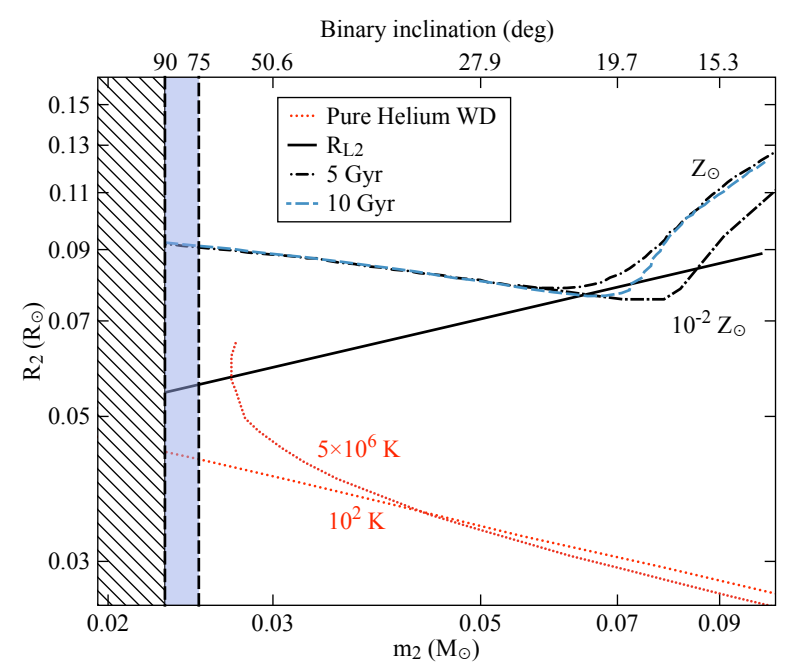

Fig. 5. Radius-mass plane showing the size constraints on the companion star Roche lobe of MAXI J0911-655 (black solid line). The other curves represent theoretical mass-radius relations for hot $\left(5 \times 10^{6} \mathrm{~K}\right)$ and cold $\left(10^{2} \mathrm{~K}\right)$ pure He white dwarfs (red dotted lines), low-mass main sequence/brown dwarfs of age $5 \mathrm{Gyr}$ (black dot-dashed lines) and $10 \mathrm{Gyr}$ (cyan dashed line) for solar and sub-solar metallicity abundances. Top $x$-axes represents the corresponding binary inclination angle in degrees assuming a NS of $1.4 M_{\odot}$. The hatched region represents the constraints on the companion mass from the binary mass function, while the blue shaded area represents the mass constraints for inclination angles between 75 and 90 degrees.

(0.4-100 keV) unabsorbed flux of the source varies from $\sim 4.7 \times$ $10^{-10} \mathrm{erg} \mathrm{s}^{-1} \mathrm{~cm}^{-2}$ (Obs.1) to $\sim 3.4 \times 10^{-10} \mathrm{erg} \mathrm{s}^{-1} \mathrm{~cm}^{-2}$ (Obs.2) after almost a month of outburst decay (see Fig. 1), which implies a bolometric luminosity of $\sim 5.1 \times 10^{36} \mathrm{erg} \mathrm{s}^{-1}$ and $\sim 3.7 \times 10^{36} \mathrm{erg} \mathrm{s}^{-1}$, respectively (assuming the source located at $9.5 \mathrm{kpc}$; Watkins et al. 2015). Considering $\tau=150$ days as a good proxy for the outburst duration and assuming an outburst mean luminosity of the order of $\bar{L}=4.5 \times 10^{36} \mathrm{erg} \mathrm{s}^{-1}$ (mean value between Obs.1 and Obs.2), we can estimate the amount of matter accreted into the NS during the outburst as:

$\Delta M_{\mathrm{acc}} \simeq 0.3 \times 10^{-10} \bar{L}_{36} \tau_{150} R_{1,10} m_{1,1.4}^{-1} M_{\odot}$,

where $\bar{L}_{36}$ is the mean bolometric luminosity in units of $10^{36} \mathrm{erg} \mathrm{s}^{-1}, R_{1,10}$ and $m_{1,1.4}$ are the NS radius in units of $10 \mathrm{~km}$ and the NS mass in units of $1.4 M_{\odot}$, respectively. Considering that in binary systems with very low-mass companions the mass transfer via the Roche-lobe overflow is driven by angular momentum loss from GR (see e.g. Verbunt 1993), we can write the long-term mass transfer rate as:

$\dot{M}_{\mathrm{GR}} \simeq 6.3 \times 10^{-11} m_{1,1.4}^{2 / 3} m_{2,0.05}^{2} P_{\mathrm{orb}, \mathrm{h}}^{-8 / 3} M_{\odot} / \mathrm{yr}$,

where $m_{2,0.05}$ represents the companion star mass in units of $0.05 M_{\odot}$ and for which we assume that the companion star is well described by the relation $R_{2} \propto M_{2}^{-1 / 3}$ suited for degenerate or fully convective stars. Substituting the two sets of mass values for the companion discussed in the previous paragraph, we estimate $\dot{M}_{\mathrm{GR}} \simeq 4.6 \times 10^{-11} M_{\odot} / \mathrm{yr}$ (for a hot pure helium white dwarf companion star) and $\dot{M}_{\mathrm{GR}} \simeq 3.8 \times 10^{-10} M_{\odot} / \mathrm{yr}$ (assuming an old brown dwarf companion star). If all the matter transferred from the donor is accreted into the NS (conservative mass transfer), we can combine the two estimates of the mass accretion rate with Eq. (7) to infer the amount of time required to transfer the matter accreted on the compact object and responsible for the observed outburst of MAXI J0911-655. In 
analogy with the transient nature of the other known AMXPs, the inferred time interval could also be interpreted as an approximate estimate of the outburst recurrence time. We obtain a time period of $\sim 3 \mathrm{yr}$ for a helium white dwarf companion star, and $\sim 0.3 \mathrm{yr}$ in the case of a brown dwarf companion star. If this is correct, even in the less favourable scenario, the source should show and it should have shown outburst episodes on the timescales of several years. The lack of observed X-ray outbursts from MAXI J0911-655 in the past $10 \mathrm{yr}$, regardless of the presence of high sensitivity, all sky monitor systems (that indeed discovered the source as soon as it went in outburst), such as the Swift BAT, reveal inconsistencies in the scenario pictured before. An intriguing, although highly speculative at this stage, way out to reconcile the expected and observed mass transfer requires substantial mass losses during the transfer between the donor star and the compact object (non-conservative mass transfer) as suggested for the AMXP SAX J1808.4-3658 (see Di Salvo et al. 2008; Burderi et al. 2009, for more details). In line with this hypothesis, we note that the observed absorption column density $\left(N_{\mathrm{H}} \simeq 0.27 \times 10^{22} \mathrm{~cm}^{-2}\right)$ is significantly larger than the averaged mapped value in the direction of the globular cluster NGC $2808\left(0.13 \times 10^{22} \mathrm{~cm}^{-2}\right.$; Dickey \& Lockman 1990; Kalberla et al. 2005). This discrepancy, already reported for the AMXP SAX J1808.4-3658 (Papitto et al. 2009), could, in principle, indicate the presence of additional neutral absorbers in the proximity of the source, in agreement with the "radio-ejection" scenario in which radiation pressure from the rotation-powered radio pulsar prevents Roche-lobe overflow with consequent losses of matter (see Burderi et al. 2002; Di Salvo et al. 2008, for more details on the model).

Acknowledgements. We thank the anonymous referee for helpful comments and suggestions that improved the paper. We thank N. Schartel for providing us with the possibility to perform the ToO observation in the Director Discretionary Time, and the XMM-Newton team for the technical support. We also use Director's Discretionary Time on NUSTAR, for which we thank Fiona Harrison for approving and the NUSTAR team for the technical support. We acknowledge financial contribution from the agreement ASI-INAF I/037/12/0. The High-Energy Astrophysics Group of Palermo acknowledges support from the Fondo Finalizzato alla Ricerca (FFR) 2012/13, project N. 2012- ATE-0390, founded by the University of Palermo. A. S. would like to thank F. Pintore for support and useful discussions. A.P. acknowledges support via an EU Marie Skodowska-Curie Individual fellowship under contract No. 660657-TMSP-H2020-MSCA-IF-2014 and the International Space Science Institute (ISSI) Bern, which funded and hosted the international team "The disk-magnetosphere interaction around transitional millisecond pulsars". N.R. acknowledges support from a Dutch NWO Vidi award A.2320.0076, Spanish grants AYA2015-71042- P and SGR20141073, and the European COST Action MP1304 (NewCompstar).

\section{References}

Alpar, M. A., Cheng, A. F., Ruderman, M. A., \& Shaham, J. 1982, Nature, 300, 728

Altamirano, D., Casella, P., Patruno, A., Wijnands, R., \& van der Klis, M. 2008 ApJ, 674, L45

Altamirano, D., Patruno, A., Heinke, C. O., et al. 2010, ApJ, 712, L58

Anders, E., \& Grevesse, N. 1989, Geochim. Cosmochim. Acta, 53, 197

Archibald, A. M., Stairs, I. H., Ransom, S. M., et al. 2009, Science, 324, 1411

Archibald, A. M., Bogdanov, S., Patruno, A., et al. 2015, ApJ, 807, 62

Balucinska-Church, M., \& McCammon, D. 1992, ApJ, 400, 699

Barthelmy, S. D., Barbier, L. M., Cummings, J. R., et al. 2005, Space Sci. Rev., 120, 143

Bassa, C. G., Patruno, A., Hessels, J. W. T., et al. 2014, MNRAS, 441, 1825

Bozzo, E., Pjanka, P., Romano, P., et al. 2016, A\&A, 589, A42

Bult, P., Patruno, A., \& van der Klis, M. 2015, ApJ, 814, 138
Burderi, L., \& Di Salvo, T. 2013, Mem. Soc. Astron. It., 84, 117 Burderi, L., Di Salvo, T., Stella, L., et al. 2002, ApJ, 574, 930

Burderi, L., Riggio, A., di Salvo, T., et al. 2009, A\&A, 496, L17

Casella, P., Altamirano, D., Patruno, A., Wijnands, R., \& van der Klis, M. 2008, ApJ, 674, L41

Chabrier, G., \& Baraffe, I. 2000, ARA\&A, 38, 337

Chabrier, G., Baraffe, I., Leconte, J., Gallardo, J., \& Barman, T. 2009, in 15th Cambridge Workshop on Cool Stars, Stellar Systems, and the Sun, ed. E. Stempels, AIP Conf. Ser., 102, 1094

Chou, Y., Chung, Y., Hu, C.-P., \& Yang, T.-C. 2008, ApJ, 678, 1316

Courvoisier, T., Walter, R., Beckmann, V., et al. 2003, A\&A, 411, L53

de Martino, D., Falanga, M., Bonnet-Bidaud, J.-M., et al. 2010, A\&A, 515, A25 de Oña Wilhelmi, E., Papitto, A., Li, J., et al. 2016, MNRAS, 456, 2647

Deeter, J. E., Boynton, P. E., \& Pravdo, S. H. 1981, ApJ, 247, 1003

Deloye, C. J., \& Bildsten, L. 2003, ApJ, 598, 1217

Di Salvo, T., Burderi, L., Riggio, A., Papitto, A., \& Menna, M. T. 2008, MNRAS, 389,1851

Dickey, J. M., \& Lockman, F. J. 1990, ARA\&A, 28, 215

Evans, P. A., Beardmore, A. P., Page, K. L., et al. 2009, MNRAS, 397, 1177

Falanga, M., Bonnet-Bidaud, J. M., Poutanen, J., et al. 2005a, A\&A, 436, 647

Falanga, M., Kuiper, L., Poutanen, J., et al. 2005b, A\&A, 444, 15

Frank, J., King, A. R., \& Raine, D. J. 2002, Accretion power in astrophysics, 3rd edn. (Cambridge University Press)

Galloway, D. K., Chakrabarty, D., Morgan, E. H., \& Remillard, R. A. 2002, ApJ, 576, L137

Gavriil, F. P., Strohmayer, T. E., Swank, J. H., \& Markwardt, C. B. 2007, ApJ, 669, L29

Gierliński, M., \& Poutanen, J. 2005, MNRAS, 359, 1261

Homan, J., Sivakoff, G., Pooley, D., et al. 2016, ATel, 8971, 1

Kaaret, P., Morgan, E. H., Vanderspek, R., \& Tomsick, J. A. 2006, ApJ, 638, 963

Kalberla, P. M. W., Burton, W. B., Hartmann, D., et al. 2005, A\&A, 440, 775

Kennea, J. A., Evans, P. A., Beardmore, A. P., et al. 2016, ATel, 8884, 1

Kirsch, M. G. F., Mukerjee, K., Breitfellner, M. G., et al. 2004, A\&A, 423, L9

Krimm, H. A., Markwardt, C. B., Deloye, C. J., et al. 2007, ApJ, 668, L147

Lebrun, F., Leray, J. P., Lavocat, P., et al. 2003, A\&A, 411, L141

Linares, M., Wijnands, R., van der Klis, M., et al. 2008, ApJ, 677, 515

Lund, N., Budtz-Jørgensen, C., Westergaard, N. J., et al. 2003, A\&A, 411, L231

Lyne, A. G., \& Graham-Smith, F. 1990, Pulsar astronomy (Cambridge University Press)

Madsen, K. K., Harrison, F. A., Markwardt, C. B., et al. 2015, ApJS, 220, 8

Markwardt, C. B., Swank, J. H., Strohmayer, T. E., in 't Zand, J. J. M., \& Marshall, F. E. 2002, ApJ, 575, L21

Massari, D., Fiorentino, G., McConnachie, A., et al. 2016, A\&A, 586, A51

Mihara, T., Nakajima, M., Sugizaki, M., et al. 2011, PASJ, 63, S623

Papitto, A., Menna, M. T., Burderi, L., di Salvo, T., \& Riggio, A. 2008, MNRAS, 383, 411

Papitto, A., Di Salvo, T., D’Aì, A., et al. 2009, A\&A, 493, L39

Papitto, A., D'Aì, A., Di Salvo, T., et al. 2013a, MNRAS, 429, 3411

Papitto, A., Ferrigno, C., Bozzo, E., et al. 2013b, Nature, 501, 517

Papitto, A., de Martino, D., Belloni, T. M., et al. 2015, MNRAS, 449, L26

Patruno, A., \& Watts, A. L. 2012, ArXiv e-prints [arXiv: 1206.2727]

Patruno, A., Altamirano, D., Hessels, J. W. T., et al. 2009a, ApJ, 690, 1856

Patruno, A., Rea, N., Altamirano, D., et al. 2009b, MNRAS, 396, L51

Patruno, A., Altamirano, D., \& Messenger, C. 2010a, MNRAS, 403, 1426

Patruno, A., Hartman, J. M., Wijnands, R., Chakrabarty, D., \& van der Klis, M. 2010b, ApJ, 717, 1253

Patruno, A., Archibald, A. M., Hessels, J. W. T., et al. 2014, ApJ, 781, L3

Pintore, F., Sanna, A., Di Salvo, T., et al. 2014, MNRAS, 445, 3745

Riggio, A., di Salvo, T., Burderi, L., et al. 2007, MNRAS, 382, 1751

Riggio, A., Di Salvo, T., Burderi, L., et al. 2008, ApJ, 678, 1273

Riggio, A., Burderi, L., di Salvo, T., et al. 2011, A\&A, 531, A140

Roy, K., Saha, T., \& Chatterjee, P. 2014, Ap\&SS, 349, 745

Sanna, A., Burderi, L., Riggio, A., et al. 2016, MNRAS, 459, 1340

Serino, M., Tanaka, K., Negoro, H., et al. 2016, ATel, 8872, 1

Stappers, B. W., Archibald, A. M., Hessels, J. W. T., et al. 2014, ApJ, 790, 39

Tudor, V., Bahramian, A., Sivakoff, G., et al. 2016, ATel, 8914, 1

Ubertini, P., Lebrun, F., Di Cocco, G., et al. 2003, A\&A, 411, L131

Verbunt, F. 1993, ARA\&A, 31, 93

Watkins, L. L., van der Marel, R. P., Bellini, A., \& Anderson, J. 2015, ApJ, 812, 149

Welsh, W. F., Robinson, E. L., \& Young, P. 2000, AJ, 120, 943

Wijnands, R., \& van der Klis, M. 1998, Nature, 394, 344 